\title{
J-integral Calculation by Using Collocation Method and Elasto-Viscoplastic Theory
}

C. W. WOO, Y. K. CHEUNG* and Y. H. WANG* Department of Mechanical Engineering, University of Hong Kong, Pokfulam Road, Hong Kong

*Department of Civil and Structural Engineering, University of Hong Kong, Pokfulam Road, Hong Kong

\section{ABSTRACT}

A combination of collocation method and elasto-viscoplastic theory is used to calculate J-integral of internal crack specimens. The results obtained

\section{INTRODUCTION}

The elasto-viscoplastic theory can be used as a unified numerical solution approach for visco-plasticity, plasticity and creep problems in solids [1], is identical to the corresponding conventional static elasto-plastic solution, for instance. On the other hand, boundary collocation method has been found to be a simple, effective and accurate tool of analysis for calculating the stress intensity factors of fracture specimens. It has been shown that the combination of the elasto-viscoplastic theory and the

collocation method provides a useful approach to the solution of a series of elasto-plastic fracture problems. In this paper, the proposed method is used to calculate the J-integral value of center-cracked and slant-cracke plates. Numerical examples show that this method offers good accuracy and requires less computation time.

BASIC FORMULATION

\section{(1) Theory of elasto-viscoplasticity}

For elasto-plastic solids, the total strain, $\varepsilon$, can be separated into elastic, $\varepsilon_{e}$, and viscoplastic, $\varepsilon_{\mathrm{vp}}$, components. The relation is expressed

$$
\varepsilon=\varepsilon_{\mathbf{e}}+\varepsilon_{\mathrm{vp}}
$$

The total stress depends on the elastic strain component according to 


$$
\sigma=D \varepsilon_{\mathbf{e}}=\mathrm{D}\left(\varepsilon-\varepsilon_{\mathrm{vp}}\right)
$$

where $D$ is the elastic matrix.

The viscoplastic flow rule is

$$
\dot{\varepsilon}_{\mathrm{vp}}=\gamma<\Phi(\mathrm{F})>\frac{\partial \mathrm{F}}{\partial \sigma}
$$

herect to time, $\gamma$ is a fluidity where (.) represents differentiation with rate, and $\Phi(F)$ is a positive parameter controlling the plastion

$$
\langle\Phi(F)\rangle=\left\{\begin{array}{cr}
\Phi(F) & \text { for } F>0 \\
0 & F \leq 0
\end{array}\right.
$$

A simple form of $\Phi(F)$ adopted in this paper is

$$
\Phi(F)=\frac{F}{\sigma_{S}}
$$

列 where $\mathrm{F}$ is the Mises yield function, an in a time interval $\Delta t_{n}=t_{n+1}-t_{n}$ can be The strain increment $\Delta\left(\varepsilon_{\mathrm{vp}}\right) \mathrm{n}$

$$
\Delta\left(\varepsilon_{\mathrm{vp}}\right)_{\mathrm{n}}=\left(\dot{\varepsilon}_{\mathrm{vp}}\right)_{\mathrm{n}} \Delta \mathrm{t}_{\mathrm{n}}
$$

According to eq. (2), the stress increment $\Delta \sigma_{n}$ is

$$
\begin{aligned}
\Delta \sigma_{\mathrm{n}} & =\mathrm{D}\left[\Delta \varepsilon_{\mathrm{n}}-\Delta\left(\varepsilon_{\mathrm{vp}}\right)_{\mathrm{n}}\right] \\
& =\Delta\left(\sigma_{\mathrm{e}}\right)_{\mathrm{n}}-\Delta\left(\sigma_{\mathrm{vp}}\right)_{\mathrm{n}}
\end{aligned}
$$

and the total stress at time $t_{n+1}$ is

$$
\sigma_{n+1}=\sigma_{n}+\Delta \sigma_{n}
$$

eq. (6), the viscoplastic strain increment can be calculated, When From eq. (6), the viscoplastic plastic problems are to be analyzed by the any value but must satisfy the important, and $\gamma$ can be take as 14$]$.

\section{(2) Collocation Method}

Using eq. (2), stresses in plastic condition can be written in tensor

$$
\sigma_{i j}=\lambda \delta_{i j} \varepsilon_{k k}+2 G \varepsilon_{i j}-\sigma_{i j}
$$

where

$$
\sigma_{i j}^{\mathrm{vp}}=\lambda \delta_{i j} \varepsilon_{\mathrm{kk}}^{\mathrm{vp}}+2 \mathrm{G} \varepsilon_{i j}^{\mathrm{vp}}
$$

Sutstituting (9) into equilibrium equation, yields

$$
(\lambda+G) u_{j, j i}+G_{i, j j}+f_{i}=0
$$

where

$$
f_{i}=x_{i}-\sigma_{i j, j}^{v p}
$$

(11) has the same form as that of elasticity, and the only difference is that $f_{i}$ here depends not only on the body force, but also on the plastic deformation.

Similarly, by substituting eq. (9) into the stress boundary equation, then

$$
\left[\lambda \delta_{i j} u_{k, k}+G\left(u_{i, j}+u_{j, i}\right)\right] n_{j}=F_{i}
$$

where

$$
F_{i}=P_{i}+\sigma_{i j} n_{j}
$$

Here $F_{i}$ is dependent on both the loading and the plastic deformation.

Suppose the displacement $u_{i}$ has the approximate expression

$$
\mathrm{u}_{\mathrm{i}} \approx \tilde{\mathrm{u}}_{\mathrm{i}}=\sum_{\mathrm{k}=1}^{\mathrm{n}} \mathrm{c}_{\mathrm{k}} \psi_{\mathrm{k}}
$$

where $\psi_{1}, \ldots, \psi_{k}$ are $k$ appropriately chosen functions, the coefficients $c_{k}$ are to be determined by collocation method.

Substituting eq. (15) into eqs. (11), (13) and displacement conditon, they will result in the residuals for the equilibrium equation, stress and displacement boundary conditions, and they are given by

$$
\left\{\begin{array}{lr}
R_{d}=(\lambda+G) \tilde{u}_{j, j i}+G \tilde{u}_{i, j j}+f_{i} & \text { in domain } \\
R_{S}=\left[\lambda \delta_{i j} \tilde{u}_{k, k}+G\left(\tilde{u}_{i, j}+\tilde{u}_{j, i}\right)\right] n_{j}-F_{i} & \text { on stress boundary } \\
R_{u}=\tilde{u}_{i}-\bar{u}_{i} & \text { on displacement boundary }
\end{array}\right.
$$

or in matrix form

$$
\{R\}=[K]\{C\}-\left\{F^{\prime}\right\}
$$

By using a least square concept, a set of equations can be obtained by By using a least square of the residuals with respect to the parameters $\{\mathrm{C}\}$ Thereafter, the coefficients of displacement function can be determined.

\section{(3) Displacement Functions}

The first step in the solution by the elasto-viscoplastic method is the calculation of elastic stress field. If some collocation points are found to have been yielded, stress relaxation can then be carried out. It is therefore necessary to select suitable displacement function $\psi_{\mathrm{k}}$ in eq. (15). for center-cracked plate problems, some stress functions which satisfy the harmonic equation in the domain are used to calculate the stress intensity factor by the boundary collocation method $[5,6]$. The corresponding displacement functions may then be taken as the basic part for $\psi_{k}$ in eq. (15).

Taking into account that stress relaxation must be carried out on both the domain and the boundary, a complementary part of the displacement function in the form of a power series of the $\mathrm{x}$ and $\mathrm{y}$ coordinates have to be added
This is used for equilibrium corrections when a point is under plastic

According to Ref. [6], the complex stress funcitons $\phi(z)$ and $\omega(z)$ may be given as 


$$
\left\{\begin{array}{l}
\phi(z)=\sqrt{z^{2}-a^{2}} \underset{k=1}{M} E_{k} z^{2 k-2}+\sum_{k=1}^{M} F_{k} z^{2 k-1} \\
\omega(z)=\sqrt{z^{2}-a^{2}} \sum_{k=1}^{M} E_{k} z^{2 k-2}-\sum_{k=1}^{M} F_{k} z^{2 k-1}
\end{array}\right.
$$

where $a$ is half crack length, and $E_{K}, F_{k}$ are undetermined coefficients. where a i.e.

$$
\left.\begin{array}{l}
\Xi_{k}=A_{k}+i B_{k} \\
F_{k}=C_{k}+i D_{k}
\end{array}\right\}
$$

The solution of the linear elastic plane problems may be obtained by the formulae[7]

$$
\left\{\begin{array}{l}
\sigma_{x}+\sigma_{y}=4 \operatorname{Re} \Phi(z) \\
\sigma_{y}-i \tau_{x y}=\Phi(z)+\Omega(\bar{z})+(z-\bar{z}) \overline{\Phi^{\prime}(z)} \\
u+i v=\frac{1}{2 G}[k \phi(z)-\omega(\bar{z})-(z-\bar{z}) \overline{\Phi(z)}]
\end{array}\right.
$$

where

$$
\Phi(z)=\phi^{\prime}(z), \Omega(z)=\omega^{\prime}(z)
$$

From eq. (17), $\Phi(\mathrm{z}), \Omega(\mathrm{z})$ and $\Phi^{\prime}(\mathrm{z})$ can be determined, and the corresponding stresses and displacements can be calculated by eq. (19). The displacement functions calculated by eq. (19) are now taken as the basic part of the approximate expression inarity, and satisfy the harmonic equation and symmetric condition.

The complementary part of the displacements, i.e. the non-harmonic functions, is assumed as follows

$$
\left\{\begin{array}{l}
u=\sum_{i=1}^{N} \sum_{j=1}^{L} c_{i j} x^{2 i} y^{2 j-1} \\
v=\sum_{i=1}^{N} \sum_{j=1}^{L} d_{i j} x^{2 i-1} y^{2 j}
\end{array}\right.
$$

The combined form of displacement functions are taken as $\tilde{u}$ in eq. (15). They are then substituted into the equilibrium equations or boundary conditions at the collocation points, then the residual equation can be obtained. By using least square method, a set of equations including the 作 the following matrix form

$$
[\mathrm{K}]\{\mathrm{C}\}=\left\{\mathrm{F}^{\prime}\right\}
$$

where $[\mathrm{K}]$ matrix can be determined from the coordinates of collocation points, $\{C\}$ is the coefficient vector $\left\{A_{k}, B_{k}, C_{k}, D_{k}, c_{i j}, d_{i j}\right\}^{T}$ which remains
to be solved, $\left\{F^{\prime}\right\}$ is the vector depending on boundary conditions and plastic deformations, and can be expressed as

$$
\left\{F^{\prime}\right\}=\left\{\begin{array}{l}
-\sigma_{i j, j} \\
P_{i}+\sigma_{i j} \cdot n_{j} \\
u_{i}
\end{array}\right\}
$$

During the first calculation step, the viscoplastic stresses and their derivatives are assumed to be zero, $\left\{\mathrm{F}^{\prime}\right\}$ can be determined by the boundary conditions by eq. (22). If the effective stresses $\sigma_{i}$ at some points are Rreater than the yield stress $\sigma_{S}$, $\sigma_{i j}^{\mathrm{vp}}$ are calculated according to eqs. (6) (8), and $\sigma_{j}{ }_{j}, j$ by finite difference method. Hence $\left\{F^{\prime}\right\}$ is obtained by eq. all points are no greater than $\sigma_{\mathrm{s}}$. The whole iterative procedure is repeated again with each load increment until the final loading is reached.

\section{(4) J-Integral}

It has been shown by Rice[8] that the following integral quantity is path independent:

$$
J=\int_{\Gamma}\left(w d y-T \frac{\partial u}{\partial x} d s\right)
$$

where $\mathrm{T}$ is the traction vector on a plane defined by the outward drawn normal, $u$ is the displacement vector, $W$ is the strain energy density and the tion is taken normal to the crack line.

When the stresses, strains and displacements have been solved, J-integral can be calculated along a given path. For a general elasto-plastic case, W

$$
w=w^{e}+w^{P}
$$

where $\mathrm{w}^{\mathrm{e}}$ is given by

$$
\text { we }=\frac{1}{2} \sigma_{i j}\left(\varepsilon_{i j}\right)^{e}
$$

in which $\left(\varepsilon_{i j}\right)^{\mathrm{e}}$ denotes the elastic components of strain. The plastic work
contribution is given by $[3]$

$$
\mathrm{W}^{\mathrm{P}}=\int_{0}^{\varepsilon_{i}^{\mathrm{P}}}{ }^{\sigma_{i} \mathrm{~d} \varepsilon_{i}^{\mathrm{P}}}
$$

where $\sigma_{i}$ is the effective stress, where $\sigma_{i j}$ is the deviatoric stress components, and $\varepsilon_{i}^{p}$ is effective plastic strain and can be calculated by the components determined by eq. (6).

\section{CENTER-CRACKED PLATE}

In two-dimension crack problem, J-integral in eq. (23) can be written as 


$$
J=\int_{\Gamma}\left\{W d y-\left[\left(\sigma_{x} n_{x}+\tau_{x y} n_{y}\right) \frac{\partial u}{\partial x}+\left(\tau_{x y} n_{x}+\sigma_{y} n_{y}\right) \frac{\partial v}{\partial y}\right] d s\right\}
$$

For plane stress case, $\sigma_{z}=\tau_{y z}=\tau_{z x}=0$,

$$
W^{e}=\frac{1}{2 E}\left[\left(\sigma_{x}^{2}+{ }_{y}^{2}\right)-2 v \sigma_{x} \sigma_{y}+2(1+\nu) \tau_{x y}^{2}\right]
$$

and for plane strain case, $\sigma_{z}=\nu\left(\sigma_{x}+\sigma_{y}\right), \tau_{y z}=\tau_{z x}=0$,

$$
W^{e}=\frac{1}{2 E}\left[\left(1-v^{2}\right)\left(\sigma_{x}^{2}+\sigma_{y}^{2}\right)-2 v(1+v) \sigma_{x} \sigma_{y}+2(1+v) \tau_{x y}^{2}\right]
$$

The effective or equivalent plastic strain for plane problems is given by the following expression

$$
\mathrm{d} \varepsilon_{i}^{\mathrm{p}}=\frac{\sqrt{2}}{3} \sqrt{\left(\mathrm{d} \varepsilon_{\mathrm{x}}^{\mathrm{p}}-\mathrm{d} \varepsilon_{y}^{\mathrm{p}}\right)^{2}+\left(\mathrm{d} \varepsilon_{\mathrm{y}}^{\mathrm{p}}\right)^{2}+\left(\mathrm{d} \varepsilon_{\mathrm{x}}^{\mathrm{p}}\right)^{2}+\frac{3}{2}\left(\mathrm{~d} \gamma_{x y}^{\mathrm{p}}\right)^{2}}
$$

At any plastic point $d \varepsilon_{i}^{p}$ can be calculated by the stress components.

aned the above analysis, the calculation of J-integral can be performed along a given path. Because of the characterization of path-independence, it is convenience to chose the path which composed by several line segment which are parallel to the cocrdinates axes as shown in Fig. l. In the and the total J-integral is twice the value.

Along line segment $A B$ in the above figure, $n_{x}=1, n_{y}=0, d s=d y$, from eq. (27) the increment of J-integral can be calculated by

$$
J_{A B}=\int_{0}^{L}\left[\left(W^{e}+w^{P}\right)+\left(\sigma_{x} \frac{\partial u}{\partial x}+\tau_{x y} \frac{\partial v}{\partial x}\right)\right] d y
$$

Alone $B C, n_{x}=0, n_{y}=1, d y=0, d s=-d x$, then the increment becomes

$$
J_{B C}=\int_{L_{x}}^{-L}\left(\tau_{x y} \frac{\partial u}{\partial x}+\sigma_{y} \frac{\partial v}{\partial x}\right) d y
$$

Alone $C D, n_{x}=-1, n_{y}=0, d s=-d y$, the increment is

$$
\left.J_{C D}=\int_{L_{Y}}^{0}\left(w^{e}+w^{P}\right)-\left(\sigma_{x} \frac{\partial u}{\partial x}+\tau_{x y} \frac{\partial v}{\partial x}\right)\right] d y
$$

When the coefficients of the complex stress functions have been determined, When the coefficients of

the stresses and calculated by the related formula and the integral can be evaluated by the numerical quadrature formulae.

In order to compare with the existing results in Ref. [9] where FEM is used, 作 specimen are assumed in the solution. There are altogether 85 collocation points with 47 points in the domain and 38 points on calculation, every side of the integral path
In order to compare with each other, the distances of the segments $L_{x}$ and $L_{y}$ are equal to the radial distance in calculation by FEM. For different Integral path and loading, the J values by collocation method (CM) and by ratio of the path radial distance to the half crack length.

From Table 1 and Fig. 2 it can be seen that the J-values by the two methods coincide with each other generally. The difference of the average value according to a certain loading is about $5 \%$. For the path near the crack tip, J-integral values by the two methods are far less than the average value. The reason is that the deformation near the crack tip is very violent. This must influence on the conditions of J-integral. Sumpter and Turner [10] have performed elastic-plastic finite element computations (using an incremental flow rule) on a serles of center-cracked plates. The $\mathrm{J}$ contour integral calculated in the usual way was found to be path independent for intermediate and large contours (path length 0.5 a to 10a). According to the above conclusion, it is not surprised that the J-integral values of $t$ small radial distance in Table 1 or Fig. 2 are not satisfactory. Excep these cases, the stabiity of calculation and the coincidence of the two wethods will be better.

\begin{tabular}{|c|c|c|c|c|c|c|c|c|}
\hline $0 / 0_{s}$ & $\mathrm{r} / \mathrm{a}$ & 0.1712 & 0.3248 & 0.4331 & 0.6102 & 0.7480 & 0.9744 & $\begin{array}{c}\text { average } \\
\text { value }\end{array}$ \\
\hline \multirow[b]{2}{*}{0.4} & CM & 0.2397 & 0.2402 & 0.2401 & 0.2396 & 0.2390 & 0.2382 & 0.2394 \\
\hline & FEM & 0.2474 & 0.2556 & 0.2548 & 0.2558 & 0.2565 & 0.2579 & 0.2545 \\
\hline \multirow[b]{2}{*}{0.5} & $\mathrm{CM}$ & 0.4536 & 0.5109 & 0.5205 & 0.5107 & 0.5018 & 0.4874 & 0.4975 \\
\hline & FEM* & & & & & & & \\
\hline \multirow[b]{2}{*}{0.6} & CM & 0.6094 & 07.197 & 0.7593 & 0.7840 & 0.7822 & 0.7614 & 0.7361 \\
\hline & FEM & 0.7136 & 0.7645 & 0.7697 & 0.7837 & 0.7831 & 0.7904 & 0.7675 \\
\hline
\end{tabular}

In the above calculation by the collocation method, every side of the contou is divided into 10 segments. Similar calculations have also been performed for 5,15 or 20 segments, results for different segments also coincide very the 11 or the calculation by $\mathrm{CM}$ and elasto-viscoplastic theory is satisfactory.

Table 1. J $\left(\mathrm{x \sigma}_{\mathrm{S}}^{2} \mathrm{bx} 10^{-4}\right)$ values by CM and FEM

* When $\sigma / \sigma_{\mathrm{S}}=0.5$ there are no calculated data listed in Ref. [8].

\section{SLANT-CRACKED PLATE}

The calculation of J-value for mixed mode problem can be carried out as for center-cracked plate. According to the definition of J-integral and the angular period of $\pi$ with respect to the center of the crack, J-value can be integral is

$$
\mathrm{J}=\mathrm{J}_{1}+\mathrm{J}_{2}=\left(\mathrm{J}_{\mathrm{AB}^{+}} \mathrm{J}_{\mathrm{BC}^{+}} \mathrm{J}_{\mathrm{CD}}\right)+\left(\mathrm{J}_{\mathrm{A}^{\prime} \mathrm{B}^{\prime}}+\mathrm{J}_{\mathrm{B}^{\prime} \mathrm{C}^{\prime}}+\mathrm{J}_{\mathrm{C}^{\prime} \mathrm{D}^{\prime}}\right)
$$

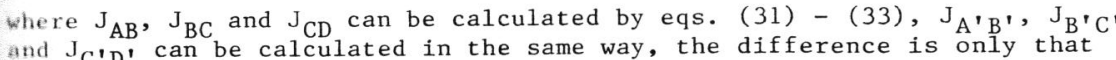


along $B^{\prime} C^{\prime}, d s=d x$.

For the mixed mode examples shown in Fig. 3, the collocation points an The the material constants are: the plastic zones are dimensions are: $h / b=2, a / b=0.2475$, $\mathrm{E}=2 \times 10^{\circ}, v=0.3, \mathrm{~s}=1$. The $\alpha=45^{\circ}$. J-integral values have $/$ a, are taken as $0.05,0.075,0.1,0.125$, $0.15,0.175,0.2$ respectively.

\section{(1) Plane Stress Case} In the first step, the stress intensity condition, the relation betwacture problem is [11]

$$
J=\frac{1}{E^{1}}\left(K_{I}^{2}+K_{I I}^{2}\right)
$$

where $E^{\prime}=E$ for plane stress case, and $E /\left(1-v^{2}\right)$ for plane strain case.

By bor method, $\mathrm{K}_{\mathrm{I}}$ and $\mathrm{K}_{\mathrm{II}}$ for the case have been calculated, By boundary collocation methe $=0.5424$. Inserting them into eq. (35), $\mathrm{K}_{\mathrm{I}} /(\sigma \sqrt{\mathrm{a}} \mathrm{a})=0.6090, \mathrm{~K}_{\mathrm{II}} /(\sigma \mathrm{d}) \mathrm{J}$-values in the different loading and paths $\mathrm{J}=0.5236 \times 10^{-5}$. The

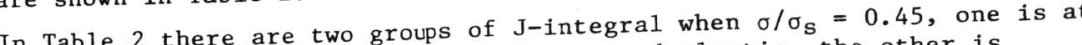
are two groups of $J$-integral whentic, the other is the first step and the deformation is assumed calculated by stress relaxation using 5 . This differs from that calculated step the average $J$-value is by eq. (35) by $2.5 \%$. From Table 2 it can the among the J-values in a certain loading path independence of J-integral for mixed mode fracture

The mixed mode plate (plane stress)

\begin{tabular}{llllllllll}
\hline \multicolumn{2}{r}{$r / \mathrm{a}$} & 0.05 & 0.075 & 0.1 & 0.125 & 0.15 & 0.175 & 0.2 & $\begin{array}{c}\text { average } \\
\text { value }\end{array}$ \\
$\sigma / \sigma_{\mathrm{s}}$ & & & & & & & & \\
\hline 0.45 & $(\mathrm{e})$ & 0.5229 & 0.5218 & 0.5198 & 0.5162 & 0.5100 & 0.5002 & 0.4853 & 0.5109 \\
& 0.6921 & 0.6870 & 0.6739 & 0.6572 & 0.6375 & 0.6149 & 0.5896 & 0.6503 \\
\hline 0.6075 & 1.034 & 1.105 & 1.114 & 1.102 & 1.078 & 1.046 & 1.007 & 1.0694 \\
\hline 0.6413 & 1.097 & 1.198 & 1.217 & 1.212 & 1.190 & 1.159 & 1.119 & 1.1703 \\
\hline 0.675 & 1.156 & 1.281 & 1.310 & 1.310 & 1.294 & 1.263 & 1.222 & 1.2623 \\
\hline 0.6856 & 1.202 & 1.351 & 1.387 & 1.388 & 1.371 & 1.338 & 1.294 & 1.3330 \\
\hline
\end{tabular}

Note: when $\sigma / \sigma_{s}=0.45, \mathrm{~J}$ ralues have been calculated at the first

$$
\text { (elastic) and }
$$

\section{(2) Plane Strain Case}

For the plane strain case J-integral has also been evaluated and shown in For the plastic calculation, the at
Is $0.5728 \times 10^{-5}$ while it is $0.5882 \times 10^{-5}$ by eq. (35) using the boundary collocation method. The difference between them is about $2.5 \%$. From the table it is also seen that the J-integral can be evaluated by the CM and be shown by the calculation using this method.

\begin{tabular}{|c|c|c|c|c|c|c|c|c|}
\hline $\int_{0}{ }^{r / a}$ & 0.05 & 0.075 & 0.1 & 0.125 & 0.15 & 0.175 & 0.2 & $\begin{array}{c}\text { average } \\
\text { value }\end{array}$ \\
\hline (e) & 0.5874 & 0.5586 & 0.5838 & 0.5793 & 0.5718 & 0.5598 & 0.4143 & 0.5728 \\
\hline $0.5(p)$ & 0.7739 & 0.7688 & 0.7556 & 0.7385 & 0.7183 & 0.6951 & 0.6691 & 0.7313 \\
\hline 0.675 & 1.152 & 1.212 & 1.215 & 1.193 & 1.171 & 1.140 & 1.099 & 1.1689 \\
\hline 0.7312 & 1.268 & 1.362 & 1.377 & 1.361 & 1.342 & 1.310 & 1.267 & 1.3267 \\
\hline 0.7736 & 1.383 & 1.511 & 1.540 & 1.529 & 1.513 & 1.482 & 1.436 & 1.4849 \\
\hline 0.7854 & 1.403 & 1.536 & 1.569 & 1.560 & 1.545 & 1.515 & 1.469 & 1.5139 \\
\hline
\end{tabular}
Table 3. J $\left(\mathrm{x}_{\mathrm{S}}^{2} \mathrm{~b} \times 10^{-5}\right.$ ) values for the mixed mode plate

when $\sigma / \sigma_{s}=0.5$, J values have been calculated at the first ste

(elastic) and the last step (plastic). Both groups are listed.

CONCLUSION

The collocation method and elasto-viscoplasstic theory is effective for (atcic studying elasto-plastic problems, his method has comparable accuracy, but rone. Whe computational time. The method requires smaller com and can be used to study other crack problems, with a intable change in displacement functions.

\section{REFERENCES}

1) Zienkiewicz O.C. and I.C. Cormeau. (1974). Visco-Plasticity, Anified Numerical Solution Approach. Int. J. Num. Meth. Engng., $8,821-845$.

(2) Perzyna, P. (1966). Fundamental Problems in Visco-Plasticity, In Recent Advances in Applied Mechanics. Academic Pres

(3) Owen, D.R.J. and E. Hinton. (1975). Finite Element in Plasticity,

4) Cormeau, I.C.(1975). Numerical Stability in Quasi-Static Elasto/ViscoPlasticity. Int. J. Num. Meth. Engng., 9, 109-127.

(1964). A Numerica ( Finite Plate. J. Basic Engng., 86, 681-684.

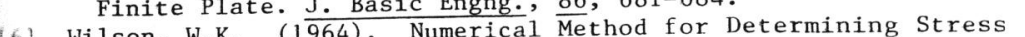
Hison, W.K. (1964). Nu Inerior Crack in a Finite Plate. J. Bas. Intensity Factors of an

Engng.', 86, 681-684. (1975) Some Basic Problems of Mathematical Theory of Elasticity. Noordhoff. 
[8] Rice J.R. and G.F. Rosengren. (1968). Plane Strain Deformation near a [8] Rice J.R. and G.F. Rosengren. (1968). Plane J. Mech. Phys. Solids, 16, 1-12. 16, D.R.J. and A.J. Fawkes. (1983). Engineering Fracture Mecha.

[9] Owen D.R.J. and A.J. Fawkes. (19ations. Pineridge Press, Swanesa. Numerical Methods and Applica. (1973). Note on the Applicability of

Int. J. Frac., 9, 320-321.

[11] Janning (1985). Advancedar. Oxford University Press.

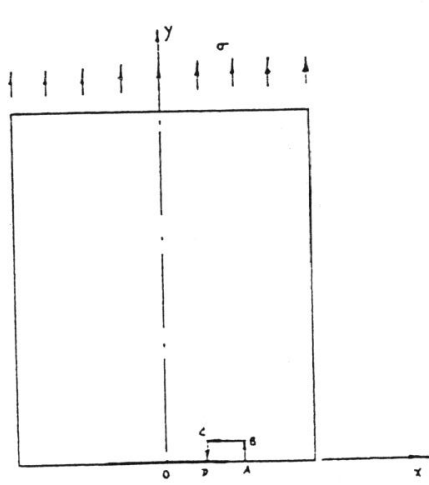

Fig. 1 Calculation path of J-integra for center-cracked plate

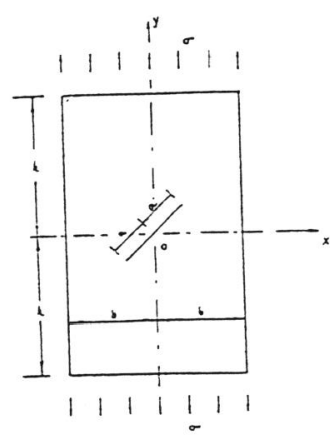

Fig. 3 Center slant cracked plate

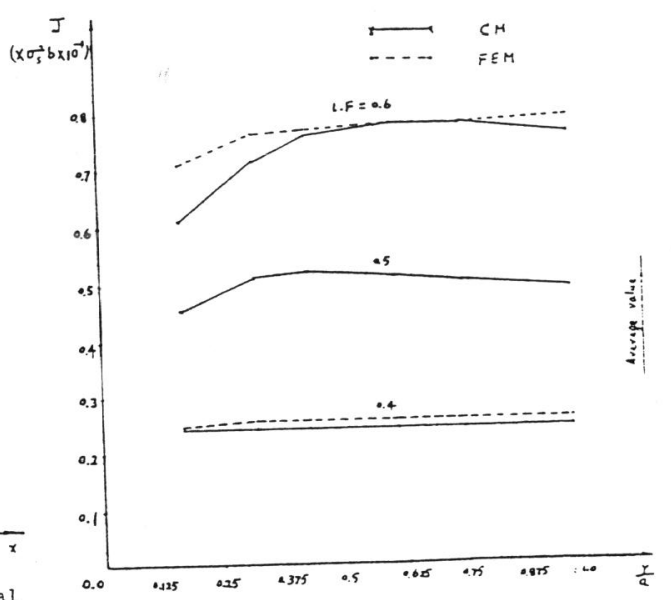

Fig. 2 Comparison of $\mathrm{J}$ values by $\mathrm{CM}$ and FEM

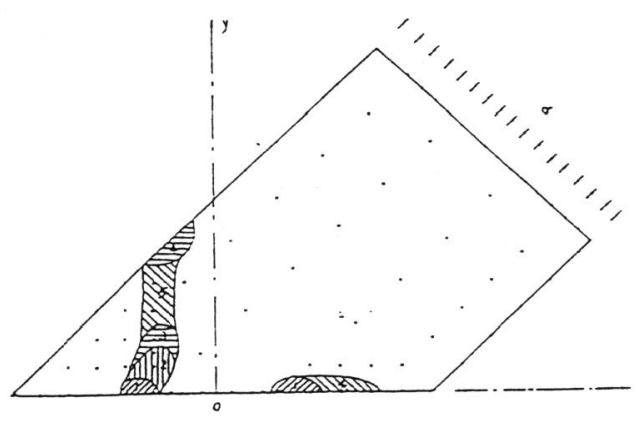

Fig. 4 The collocation points and plastic zone 